\title{
Using Both EMDR and Prescriptive Play Therapy in Adaptive Information Processing: Rationale and Essential Considerations for Integration
}

ANN BECKLEY-FOREST

\section{INTRODUCTION}

Despite the potential benefits, children are often very reluctant to participate in eye movement desensitization and reprocessing (EMDR) therapy. Children may accomplish some digestion of traumatic experiences in play therapy, a naturalistic setting in which children participate eagerly. Prescriptive play therapy for trauma involves a phase-based approach where the activities within the playroom may vary from less directive to more directive with the goal of supporting trauma exposure/trauma narrative work. Trauma-informed prescriptive play therapists guide play in the avenues that will support trauma digestion and emotional regulation. Integrating play therapy within Francine Shapiro's adaptive information processing (AIP) model with a flexible approach to the EMDR protocol holds promise in using play to enter the memory network and promote healing. This chapter establishes eight essential considerations in fully integrating EMDR in a play therapy setting and examines the idea that play may be the preferred avenue to access and reprocess with EMDR the implicit memories involved so often in complex trauma in children.

\section{TWO COMMUNITIES OF THERAPISTS WITH ONE GOAL}

A newly trained therapist, well versed in the most current understanding of how trauma and adversity affect the long-term emotional and physical health of children, sets out on a career oriented toward helping children to heal. But where to begin? How can therapists best learn to provide the kinds of experiences within treatment that will help children to feel competent, loved, and optimistic despite 
the wounds to their neurobiological development from trauma? In my career, I have had the privilege of operating within two different professional communities both focused on improving outcomes for such children-play therapy and EMDR therapy. I have developed a firm belief that cross-discipline conversation will lead to more fully integrated models and deeper healing. These communities must understand the contributions made by each respective field and ultimately develop ways of integrating on both a theoretical and practical level while remaining faithful to each approach.

\section{FOR EMDR THERAPISTS: UNDERSTANDING PLAY THERAPY AS A CLINICAL APPROACH}

The term "play therapy" broadly describes a variety of models and theoretical approaches that clinicians use to create opportunities for therapeutic change, learning, and healing among children. What sets play therapy apart from other child therapies is the intentional, not accidental or incidental, use of play. Play therapists see play as "the specific change agent" that initiates, facilitates, or strengthens the therapeutic effect. Regardless of the play therapy model, play therapists understand play itself to produce change, beyond being merely a way of making treatment more accessible or palatable (Schaefer \& Drewes, 2014). Traditionally, notions of play therapy were associated with child-centered play therapy or CCPT, which developed from the early work of Virginia Axline (1964) and was further developed by Gary Landreth (2012) and others. CCPT relies on the therapist providing an environment for expressive play in which the child's activities are noticed and supported in a calm, neutral way by a therapist who respects the child's own power in using the experimentation of play to resolve emotional difficulties. In CCPT's companion filial approach, child-parent relationship therapy (CPRT), parents are coached to provide the acceptance and reflecting statements that a play therapist would use (Landreth \& Bratton, 2019). Over time, play therapy as a clinical approach has developed to include evidence-based approaches along a continuum from child-led to therapist-led, also described as the continuum from nondirective to directive play therapy, including Adlerian play therapy (Kottman, 2013) and cognitive behavioral play therapy (Knell, 2009) among others.

The term prescriptive play therapy (Schaefer, 2011) describes a theoretical approach to play therapy that draws from a variety of modalities and approaches within play therapy in an integrated manner to develop a comprehensive assessment and treatment plan. This plan applies the healing power of play through a variety of child-led and adult-influenced activities. Prescriptive play therapy is being widely applied in the treatment of trauma, as it balances both the establishment of a CCPT relationship where posttraumatic play can emerge (Gil, 2006; Terr, 1990), with the urgent need for the therapist to direct children along the path of trauma digestion and exposure work. Phobic avoidance of trauma triggers might make a purely child-centered approach ineffective, as many clinicians who use CCPT have observed that when the child is so defended from the traumatic memories, their play in the therapy room feels superficially cheerful. Prescriptive play therapists rely heavily on a foundation of CCPT, especially in the earliest phases of treatment, to establish the relationship, increase attunement, and develop a case 
conceptualization of the child's unique needs and responses. In tandem with this free play, the prescriptive therapist would use additional directive strategies to accomplish therapeutic purposes, such as teaching self-soothing and nervous system regulation, or addressing specific therapeutic needs, such as the digestion of an overwhelming or upsetting event, using a trauma protocol such as EMDR.

Play therapy as a discipline within psychotherapy places a large emphasis on a therapeutic environment that promotes the therapeutic benefits of play in the presence of a play therapist trained to respond deliberately to the child in accordance with the specific play therapy approach. While play therapy environments do vary, a typical play therapy room offers many options for dramatic and role play, such as props, simple costumes for role playing, household items, a doctor's kit, dolls, expressive art materials, simple musical instruments, puppets, items for active play such as balls, and items that are interesting to touch and hold such as items from nature (Landreth, 2012). In addition, many play therapists also have a sand tray and an assortment of miniature figures to use in projecting "worlds" expressively in the sand, including human and animal figures, miniature versions of everyday items as well as fantasy and iconic symbols, and landscape props such as trees and bridges and other items (Homeyer \& Sweeney, 2016). Younger children tend to use the sand tray as a miniature world in which to play actively, whereas older children respond to prompts to use the sand tray and miniatures as an expressive space for projection of feelings and experiences.

However, not all play therapy approaches emphasize the provision of toys and props for projective play. Some are more game-based or focused on an interactive approach and may use few or no props, such as Theraplay ${ }^{\mathrm{TM}}$ (Jernberg \& Booth, 2001) or developmental play therapy (Brody, 1997).

Play therapists are informed by a variety of theoretical models and vary in terms of the emphasis placed on verbal expression of feelings and developing insights versus experimentation and experiences kept in the metaphor of the play with little or no explicit self-reflection. Over the past decade, increasing emphasis on establishing the research support for various play therapy approaches has led to many published studies and meta-analyses affirming the clinical efficacy of using play therapy to treat a variety of childhood mental health conditions, despite the difficulties in studying what is often a nonprotocolized and highly individualized approach to intervention (Bratton, Ray, Rhine, \& Jones, 2005; Lin \& Bratton, 2015; Ray, Armstrong, Balkin, \& Jayne, 2015).

\section{FOR PLAY THERAPISTS: UNDERSTANDING EMDR AS A CLINICAL APPROACH WITH CHILDREN}

Francine Shapiro's AIP model proposes that the integration of both positive and negative experiences into our nervous system is the healthy process by which we grow. When an acutely negative or traumatic event occurs, this information processing is sabotaged by our own neurobiological effort to cope with the trauma by isolating the related associations, images, feelings, and so forth (Shapiro, 2017). Our own phobic avoidance of reminders of the pain, fear, and shame of the traumatic experience contributes to further isolation of this network of related associations. The more severe and complex the trauma, the more elaborate this 
isolation becomes, even resulting in dissociation and the exiling of the part of self that had the traumatic experiences from present awareness (Gomez, 2012). EMDR's potency in entering this isolated memory network and enabling the client to begin the adaptive process of integration is well established in the literature (Chen et al., 2014) and has been listed as an evidence-based treatment for posttraumatic stress by Substance Abuse and Mental Health Services Administration (SAMHSA) since 2010 (SAMHSA, 2012).

In order for EMDR to be effective in helping the client to "digest" the trauma-or in AIP language, remove blocks to allow adaptive information to reach the memory network or node-we have to be able to reach and activate the memory and its related associations. The therapy has to allow the resurfacing of disturbing images, sensory data, emotions, body sensations, and the co-occurring cognitions without overwhelming clients and causing their systems to shut down. The eight phases of the EMDR protocol guide the clinician in first identifying these memory nodes or touchstone memories, then preparing the client to manage the intensity of the stored emotions and sensory data, followed by facing and digesting the upsetting elements of the trauma while using eye movements or another form of bilateral stimulation (BLS), and finally attaching more adaptive self-referential beliefs to the experience to promote full integration of this memory into the client's own narrative. EMDR emphasizes attention to the somatic experience of the trauma throughout this processing (Shapiro, 2017).

Since the earliest days of EMDR, practitioners have attempted to extend the benefits of EMDR to children (Greenwald, 1999; Tinker \& Wilson, 1999), and there is a growing body of evidence in support of the outcomes of this treatment with trauma-exposed children (Adler-Tapia \& Settle, 2008; de Roos et al., 2010; Fleming, 2012; Kemp, Drummond, \& McDermott, 2010; Moreno-Alcazar et al., 2017). In considering what AIP looks like in children versus adults, we recognize that children do the majority of their learning through action and imaginative experimentation-also known as "play"-not through the verbal reflection or even the visual imagery that is the primary portal of processing for most adults using EMDR. There is wide acceptance that the therapist must include movement, props, and other aspects of play into child-friendly EMDR. Some examples would include having the child draw images from the target memory (Adler-Tapia \& Settle, 2016; Tinker \& Wilson, 1999), using sensory experiences to evoke the safe/ calm state in the preparation phase (Gomez, 2012), using the sand tray to create a narrative of the trauma experience during reprocessing (Gomez, 2012), or using BLS to install positive experiences of attachment during baby role play (McGuiness, 2003). These authors have established the need for EMDR with children to be adapted to make EMDR more appealing as well as more comprehensible.

\section{TAKING THE NEXT STEPS IN INTEGRATION: THE EIGHT ESSENTIALS}

This chapter represents an effort to advocate for an even more intentional and systematic integration of EMDR therapy with established play therapy principles and approaches on the basis of understanding several ideas: (a) The therapeutic alliance available to children in a play therapy context, especially in CCPT, expands 
the emotionally safe space that is needed for trauma work. (b) The AIP described by Shapiro will be most fully realized in children who are actively engaged in dramatic and expressive play, as the neural networks the therapist is trying to reach are mostly available during play. Children are often already engaging in posttraumatic play if only the therapist is able to enter the metaphor, understand this communication, and invite processing. (c) A thoughtful integration of play therapy strategies with EMDR will reduce the risk of flooding children with traumatic material too soon, by building the bridge between the metaphor of the play and their own first-hand experiences gradually, staying within their ability to tolerate, even while engaging in reprocessing with EMDR.

In clinical work with children and as a trainer and consultant within both the EMDR and play therapy communities, I have struggled alongside my colleagues to articulate a comprehensive approach to integrating the best of both approaches in meeting the needs of the individual child survivors of trauma. In this context, I have developed a series of eight essential considerations for fuller integration of play therapy with EMDR therapy based on a paper previously published on this topic (Beckley-Forest, 2019; see Figure 1.1).

This working model of the eight essential components is not rigidly hierarchical but builds gradually over time, beginning with a more nondirective stance on the part of the therapist and layering in essential skills before more actively engaging the child in explicit first-person trauma work using EMDR. It is most compatible with a prescriptive play therapy approach, where the therapist flexibly uses a mixture of child-centered playtime along with child-responsive interventions from the therapist, using the lightest touch possible with these insertions and suggestions, such as wondering aloud-_"I wonder what would happen if. . . ." Using these kinds of observations in place of more didactic teaching or even gentle questioning can help shifts in thinking to be more congruent, much as the gentle language EMDR therapists would use along with interweaves in EMDR processing. In addition, prescriptive play therapists use information gained through observing and attuning to the child's play to develop a menu of play-based skill-building activities for the child and family to complement the play in which the child is already engaging. A prescriptive approach to play therapy is quite compatible with integration with the eight phases of EMDR. Paris Goodyear-Brown (2010a) proposes a model for flexibly sequential play therapy, a phase model that she now calls TraumaPlay ${ }^{\mathrm{TM}}$ (Goodyear-Brown, 2019), as an integration of directive and nondirective play therapy activities. Her model is an example of a play therapy approach, which is quite compatible with these eight essential considerations for integration of play therapy with EMDR.

To the child's own preferred play themes, the therapist must add a gradual introduction to EMDR-specific tools, props, and activities. We will also need an approach to enhancing the child's ability to self-soothe and change their state from agitated/upset to calm by using play that involves the parts of their neurobiology affiliated with social engagement and present time orientation.

A word about the eighth essential is needed, as it represents the overarching consideration of how to develop and maintain a parallel process with parents and the care-giving family system at all points during the course of treatment. Family involvement is associated with the best outcomes in child treatment in 


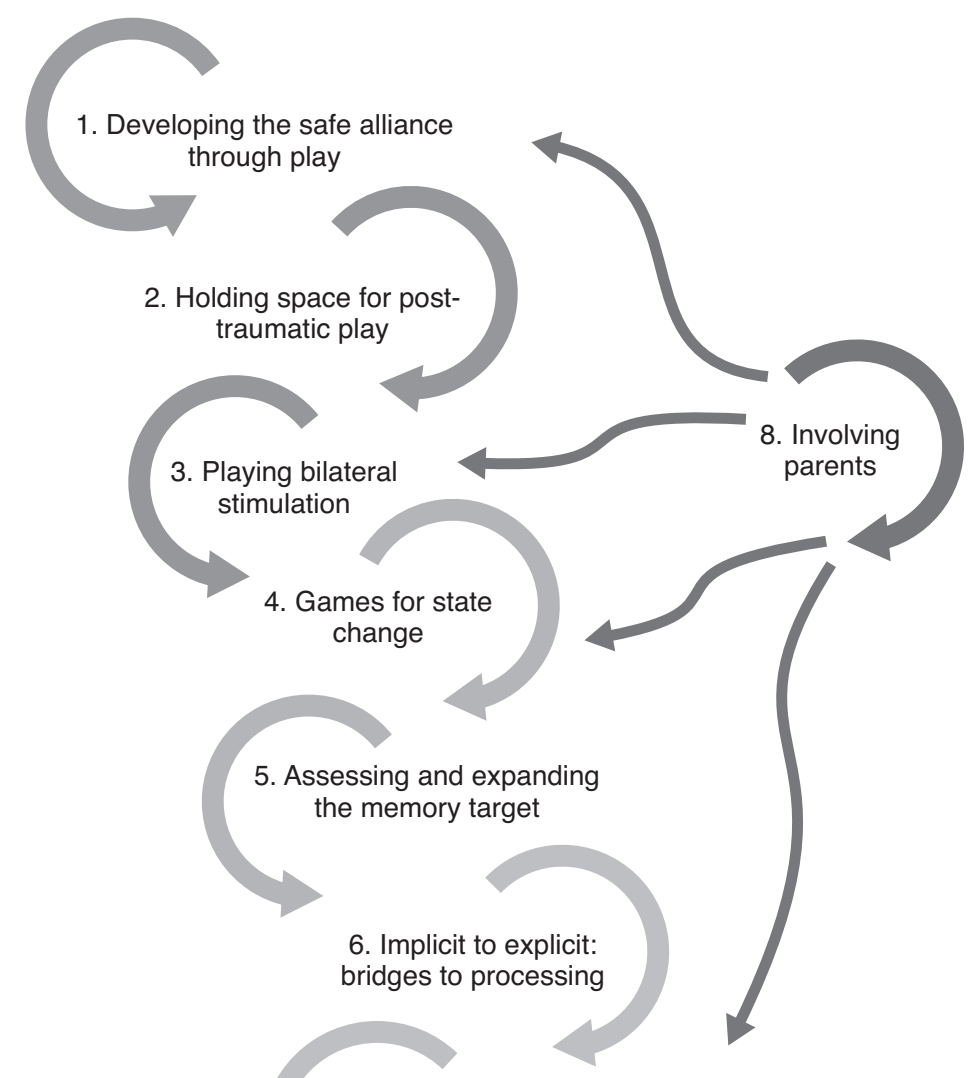

7. Ongoing reevaluation of memory targets

Figure 1.1 The Eight Essentials for Integrating Eye Movement Desensitization and Reprocessing and Prescriptive Play Therapy

both the play therapy literature (Lin \& Ray, 2015) and EMDR literature (Gomez, 2012; Lovett, 1999; Wesselman, Schweitzer, \& Armstrong, 2014).

\section{ESSENTIAL 1: SAFE ALLIANCE THROUGH PLAY}

CCPT is the foundation of the relationship between the therapist and the child in play therapy, and its unique features set it apart from other ways of being with children in treatment. In a CCPT relationship, the child is offered the opportunity to access troubling emotions and upsetting memory networks at their own pace and from a safe emotional distance. The therapist's participation in the form of tracking and reflecting statements delivered with calm, dispassionate interest is 
also regulating for the child (Landreth, 2012). Researchers describing and understanding the mechanism of how this child-centered environment helps the child to unfold and access material safely have noticed the importance of the neutrality of this arrangement in reducing the power differential in the relationship. Badenoch (2008) noted that adult directives can be experienced by the child's nervous system as a threat and activate rage in a sensitive child's limbic system (p. 302). Establishing the kind of authentic emotional safety, which enables clients to respond to therapist requests and questions honestly and without transference, has long been seen as an important process in adult therapy. In child therapy, the power differential is inherent to the relationship, as children obviously have less power than adults. Children are more likely than adults to respond to therapist directives or questions with either adult-pleasing or adult-defying behaviors, which are already part of their primary attachment repertoire. In a relationship where the therapist will ultimately be leading the child toward exposure to traumatic material, these power differences have the potential to cause great harm.

\begin{abstract}
Brenda, aged 7, is brought to treatment by her parents 6 months after a coercive sexual assault by an adolescent neighbor. She originally had coped well with parent support but over time the parents accepted her request to "stop talking about it all the time." She has become more isolated, anxious, avoidant of new situations, and has great trouble sleeping, so the parents sought treatment. She is a cooperative and highly verbal child without a significant history of disruption or adversity.
\end{abstract}

While the therapist may conclude quite readily that there is still material related to the trauma that is troubling to this child, moving too quickly to trauma work will likely backfire, even though this obedient child would possibly comply. The adult-pleasing dynamics will make it difficult for her to be honest about the intensity of her distress. If she is dissociating or flooding, the therapist may miss it, as Brenda is reading her therapist's reactions and trying to give the "right" answer. Or the opposite situation:

Sam is an 8-year-old who struggles with rules and self-regulation despite having been in a stable foster home for 3 years where he is about to be adopted. The case worker and soon-to-be adoptive parents are well informed of the impact of the trauma of removal from his biological parents 3 years earlier, and subsequent termination of contact due their inconsistency and substance abuse. They are anxious for him to receive trauma treatment to "get over" his earlier experiences and be able to flourish in his current family. He is suspicious of new people and new experiences and reacts aggressively in these instances.

While the therapist might rightly conclude that this child needs to be able to digest his own memories of neglect and removal in order to move forward, efforts to engage him directly around this kind of work will almost certainly fail, not only because he may have developed some layers of protection through dissociation, but any attempt at therapist direction will be likely met by him with suspicion leading to adult-defying behaviors in an effort to control the course of the session. 
Scenarios such as these might be very challenging in a directive or protocoldriven model of treatment, but play therapists frequently rely on CCPT to establish an egalitarian therapeutic relationship. In CCPT, the therapist invites the child to "use these things in any way that you want" and accepts the child's process without challenging it. Over a period of sessions conducted in this way, the child may at first test this dynamic and then visibly relax and become increasingly engaged in the play therapy space, constructed to elicit the full breadth of emotion and human experience. Without the need to earn the adult's approval—or establish independence by defying the norms the child sees the adult as enforcing-the play therapy space becomes a more purely projective-holding environment for the child's own ideas about the world and a safer place to experiment with the mastery of feelings and experiences, including traumatic experiences. The narrative themes that emerge in the play may contain metaphors, which reflect the child's emotional landscape, communicate about how the child views the world and their place in it, and contain sensory pieces of traumatic memories or even literal reenactments of the trauma. The autonomy of the child in the playroom is a felt experience of empowerment, which is essential to therapeutic change.

In order to help children who have been affected by adverse experience to move forward in therapy as quickly as possible, quite paradoxically the therapist should initially "slow down" and use CCPT to establish therapeutic safety. In addition to helping establish the egalitarian relationship, this child-led dynamic may allow for the emergence of play already activating the trauma memory network and providing information to the therapist about the child's experiences of the trauma, especially where verbal disclosure may be difficult, if not impossible. This material is allowed to emerge congruently, without activating the child's defenses.

\section{ESSENTIAL 2: HOLDING SPACE FOR POSTTRAUMATIC PLAY}

The term "posttraumatic play" is credited to the groundbreaking work of Lenore Terr in the 1990s. Her extensive work studying and interviewing children who had survived significant trauma led her to conclude that not only are traumaexposed children often affected by a lingering sense of terror without words, but that the "fear of further fear" will lead them to avoid accessing support from caregivers. Instead, elements of the trauma are approached in reenactive play, which she noted was often done in secret, "grim and monotonous, obsessively repeated, and may not relieve anxiety" (Terr, 1990, p 238).

Eliana Gil (2006) describes posttraumatic play as a repetitive and often rigid type of play initiated by children who are trying to "expose themselves to the literal aspects of the trauma which cause them despair" (p. 184). The AIP model would describe these elements in the play as connected to the memory node, which holds the trauma, and thus offering a possible pathway into the associated neural network. If the child is able to gradually move in and out of this processing in a dynamic way, the brain's own drive toward integration will promote healing. Gil's work within play therapy has been significant in helping with the complexities of recognizing and supporting dynamic play, which may be posttraumatic in nature, providing options for intervening to prevent the play from becoming static and potentially retraumatizing as well as using play to ground children who begin to 


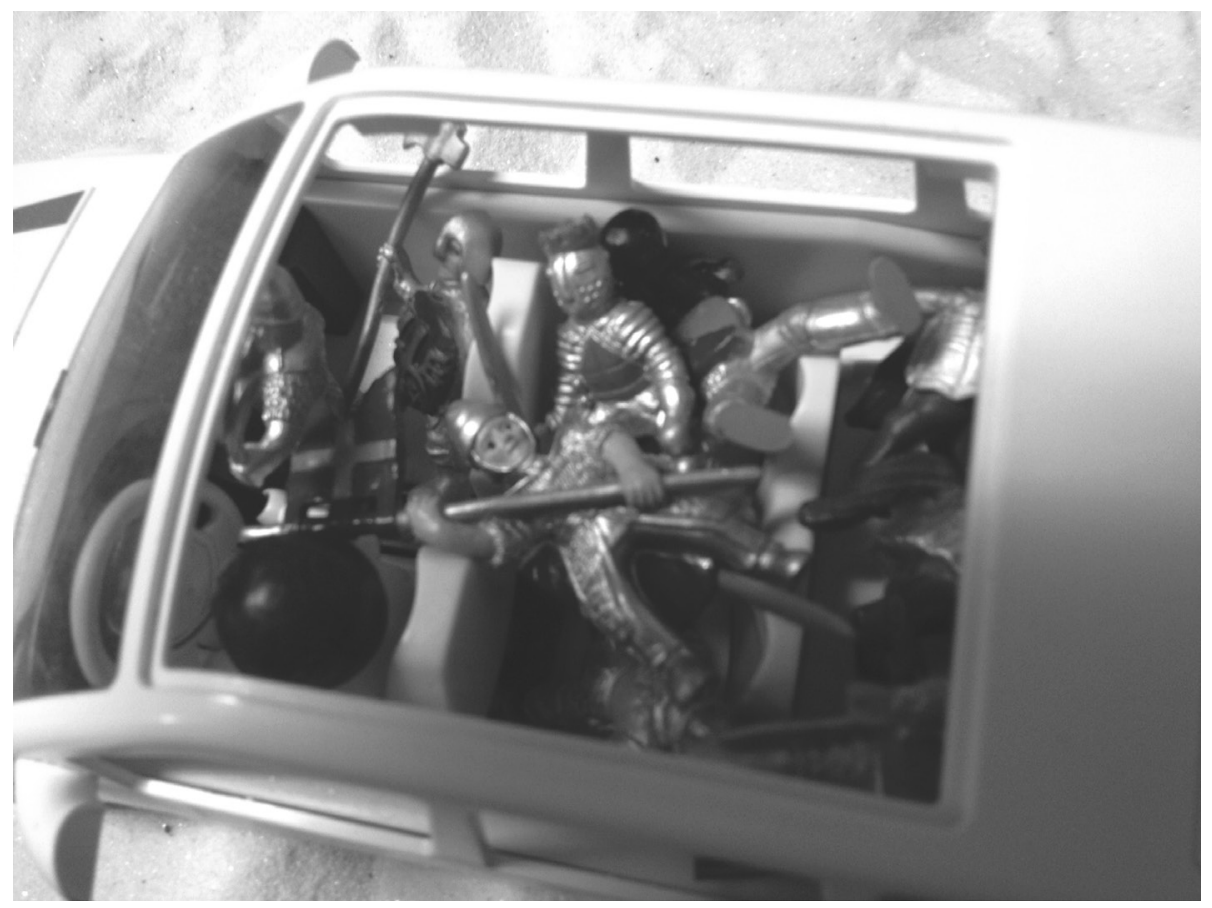

Figure 1.2 The Trauma Memory Network: "They Can't See, They Can't Get Out, They Can't Get Away"

dissociate (Gil, 2016). We can use this framework to recognize when themes in a child's play may be literal or metaphoric reenactments of traumatic experiences.

Missy was an 8-year-old child who had been adopted as a toddler. She came to therapy after a minor car accident appeared to have had a major impact on her day-to-day behavior and well-being. Offered the opportunity for CCPT in the playroom, she returned again and again to a story she created about a litter of puppies. Missy's story was enacted with the same materials each week, in the sand tray and in the dollhouse.

Missy: These are the puppies. They are being kidnapped. No one can hear how they are barking and crying. (Pitiful barking and whimpering.)

Therapist: The puppies cry and no one hears them.

Missy: Yes, and they all get stuffed in the car like this (see Figure 1.2). They can't breathe, and they can't get out. (The child herself breathed more rapidly and shallowly here, and her voice rose with anxiety.)

Therapist (intervening to keep Missy regulated, but within the metaphor): Hey puppies, I see you under there. You need a deep breath. (Therapist breathes audibly.) Can we both take a breath for the puppies?

Missy (after breathing): They are stuck in here, they can't see out (piling more figures on top). 
When children return urgently to the same materials and themes over a number of sessions, as well as when the content of the play feels upsetting versus playful and fun, we are alert to the possibility that aspects of the metaphor in the play may have begun to hold some of the trauma content (Gil, 2016). Sometimes, the therapist has to employ a fair degree of creativity and speculation in order to understand the clues that are available in the play, which can help with the gradual exposure work. In Missy's case, I began to suspect that there may have been elements of the trauma of her removal from her biological family that were available to her in the "kidnap" metaphor in the play. Once the posttrauma metaphors are unfolding in the play, we can add this information to our case conceptualization in order to begin considering how to enter this memory network to allow the flow of adaptive information. We return to this idea later when we discuss the essential consideration of expanding the target. As the therapist, I could engage in a series of speculations about the information available within the metaphor. In Missy's case, pairing the story about being kidnapped by car with elements of the trauma memory network, both the traumatic experience of being removed from her biological parents and the recent trigger experience of being trapped in her seatbelt after the car accident became a working model for me of the activation of this memory network. The distancing of the play allowed her to stay more present even as traumatic intensity emerged. The ability to approach traumatic intensity from a distance is especially important when there is a lot of fear of the memory or the memory is beyond the child's current awareness, as in early attachment trauma. When the intensity of the play is overwhelming or at risk of becoming toxic or retraumatizing to the child, a light touch with reflections and child-responsive invitations can help the play to move along dynamically, such as in the earlier example when Missy was invited to breathe during the play.

Gil's distinction between dynamic and toxic posttraumatic play is important in guiding the therapist along the continuum of in-session activities from child-centered to more directive. The autonomy of the child in the rich sensory environment of the playroom helps to mitigate the risk of children becoming overwhelmed and dissociated during posttraumatic play. If the child has internalized the belief that they have the power to decide in the playroom what to do next, they are more likely to move away from the intensity on their own to avoid being flooded by traumatic content. This possibility is one of the key reasons why the play therapy room makes an ideal setting for approaching and digesting trauma content. Another check on therapist interference considers that attempts to introduce adaptive information too soon can shut down the child's own processing, interfering with the child's opportunities for expressive release through exposure (Gil, 2016, p. 19). Trauma-exposed children already have messages in their environment from parents and others, which increase phobic avoidance of exposure to traumatic elements such as "just don't think about it" or "it wasn't so bad." When the posttraumatic play is dynamic and shows evidence of movement toward digestion, nondirective CCPT can hold the space for the play to begin the exposure and also show the therapist where directive support may be needed once the child is ready for a more explicit digesting of the experience, such as with EMDR processing added to the play.

Some children exhibit repetitively reenactive play related to the trauma, which is static and unchanging, resembling Terr's "grim and monotonous" description 
(1990). Many years ago, I worked in a Head Start setting with a young boy who, unbeknownst to anyone at the program, had witnessed a particularly frightening episode of domestic violence between his parents. Three weeks in a row, he reenacted the experience in the dollhouse, mimicking the words and actions used and grimly repeating them over and over. The play never varied in location or materials, and the emotional content was flat. This was play that did not feel at all like play or experimentation, or mastery of any kind. In addition to spurring me to action outside of the play sessions with regard to ensuring the safety of the family members, I was not able to continue as a bystander to merely track and reflect the content of this narrative session after session and needed to respond along a continuum of interventions, such as described by Gil (2016, pp. 47-48). The continuum encompasses everything from the lightest touch of verbalizing some additional descriptions and asking children to give their characters a voice, along the continuum to more directives such as asking children to change the order of events by starting the story in the middle or showing the ending first. We also can ask the child to breathe or move, or involve another medium such as drawing the story or photographing the sequence of play. I did not know EMDR in those days, but now in a similar scenario, I would add to that list the possibility of using reflections to invite moments of noticing how the characters felt or where those feelings are in their bodies as another possible way of helping move the play along in a more dynamic direction. These moments of noticing help prepare the child for EMDR processing.

\section{ESSENTIAL 3: PLAYING EMDR WITH PROP-BASED BILATERAL STIMULATION}

BLS, also called dual attention stimulation, is a unique feature of EMDR, setting it apart from other trauma-digestion approaches. In adult EMDR therapy, we emphasize eye movements following the therapist's fingers or using a light bar or other device as the primary way of delivering the BLS. With children, a wider variety of options have developed. Using magic wands, toys, or comfort items is a common strategy for the eye movements, but EMDR therapists sometimes use purely tactile BLS (Shapiro, 2017). Some options include "tappers" developed for the EMDR community. These are handheld tactile vibration devices, some with headphones, which offer alternating auditory tones. At other times, the therapist may gently tap the child with puppets, use hand claps, bang drums, or coach the child to use the "butterfly hug" (Jarero, 2002). These all represent innovations now in wide use in EMDR with children, as the need for tactile BLS arises primarily from the difficulty younger children have sustaining attention to eye movements (Tinker \& Wilson, 1999). The novelty of having a variety of methods can help engage a reluctant child. For example, having recently added some light-up drum sticks to the playroom options (inspired by Swinden, 2018), I noticed that several child clients were more engaged and interested in returning to the traumafocused narrative play that they sometimes try to avoid. Taking a kinesthetic or full-body approach inspires the use of large muscles for the BLS, here demonstrated by a young friend of mine (not a client) with a foam sword (Figure 1.3) or a ribbon wand (Figure 1.4).

Short episodes of therapist-led activities are needed to introduce this novel way of playing to the child early in treatment. These introductions to BLS or "back 


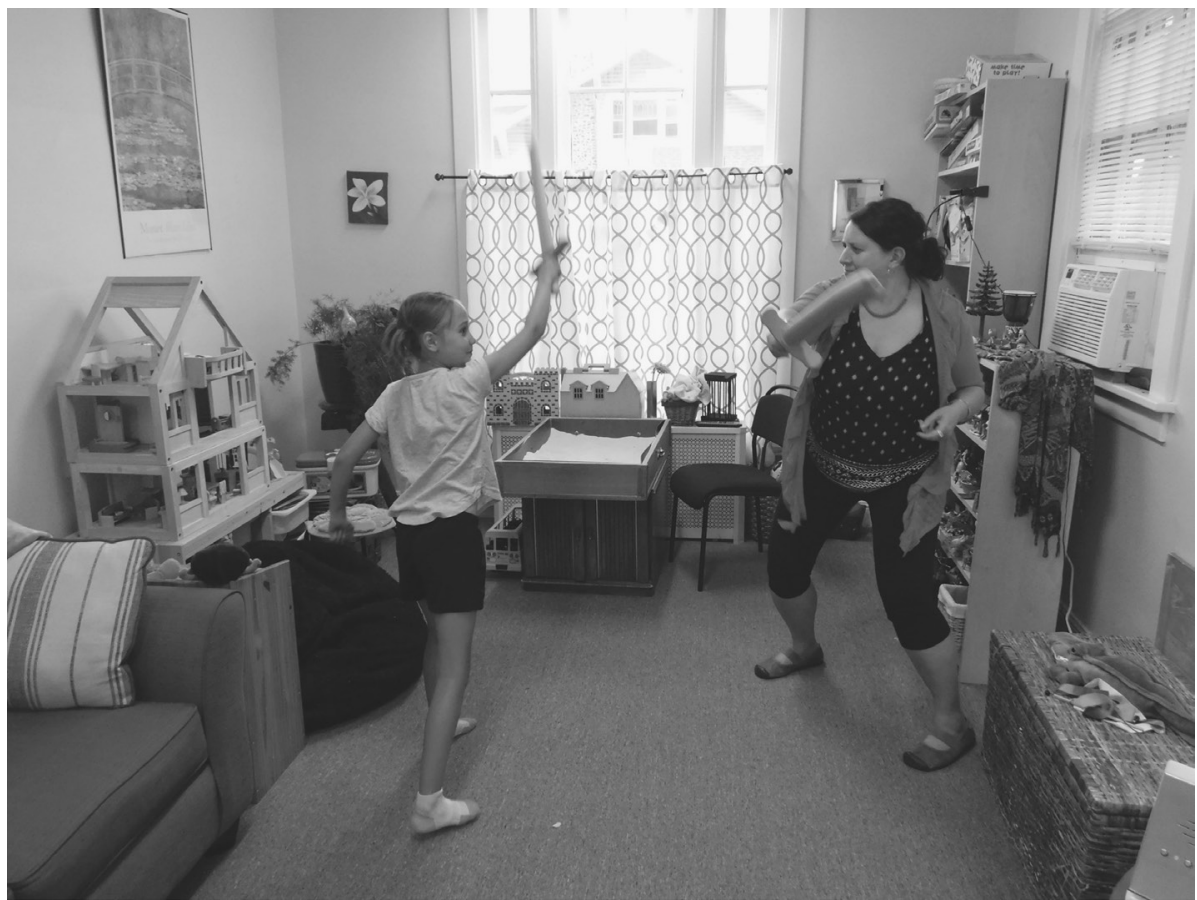

Figure 1.3 Foam Swords for Full-Body Bilateral Stimulation

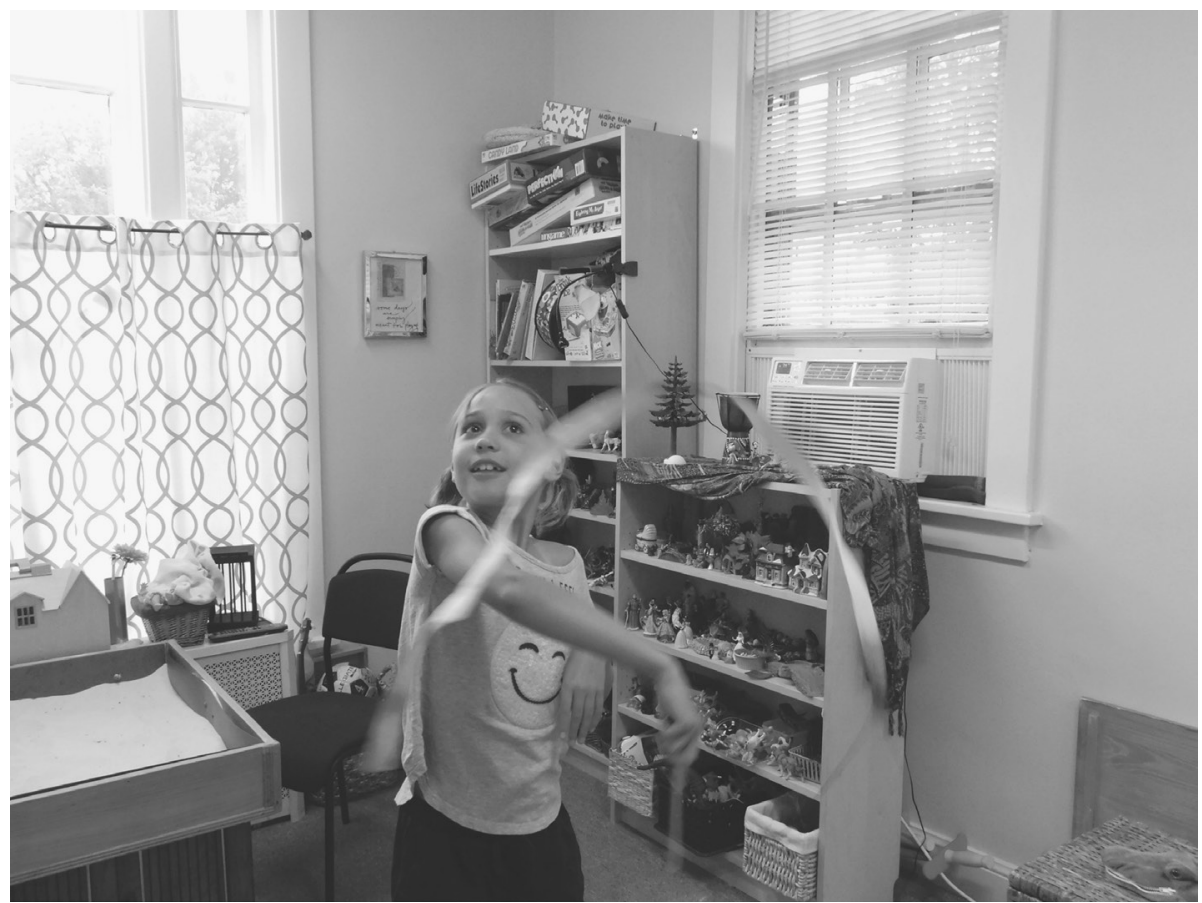

Figure 1.4 Ribbon Wand for Bilateral Stimulation 
and forth games" are an important part of the preparation phase for children. Using BLS (brief sets of 5-10 slow saccades, or back and forth passes) to install positive feelings and notice body sensations for a moment before, during, or after other play activities in the playroom sets the stage for their later use in processing. The novelty of introducing a wide variety of ways of using BLS to children eventually helps expand their capacity to sustain and reenter processing. In the earliest stages of therapy, such as during engagement with the child and family system, we can use brief directives to teach self-soothing and install positive feeling states using the BLS props and games in the context of the play therapy session. Some examples include:

Inviting children to use sand trays and expressive materials to create positive images, including constructions of safe spaces, and then using the "back and forth" movement of BLS to "find how it feels in your body/tummy/heart to see what you made."

Role playing versions of installing positive self-statements as resources. For example, with a young client with very low frustration tolerance, we developed a sword fight game using pool noodles as the swords (the full-body BLS initiated by the therapist) and the positive cognition "I can handle it" to practice and reinforce this self-talk as a resource in challenging situations. In the playfully aroused state of the sword fight, her repetition of "I can handle it" again and again helped this adaptive belief to more fully enter her nervous system where it could be more available to her during other times of hyperarousal (intervention inspired by a story in The Worry Wars, Goodyear-Brown, 2010b).

In play that is taking place within the parent-child dyad, such as in a Theraplay ${ }^{\mathrm{TM}}$ or other dyadic play therapy moments as in role-playing baby care, we can use brief sets of BLS to help the child and parent notice or tune in to the attachment experiences as an internal resource.

In fidelity to the EMDR protocol, I am not in favor of the practice of some child therapists using BLS by "letting the buzzers just run" continuously during play. The therapist should be using BLS with intention, to promote the linking of adaptive information with the memory networks, whether that information is noticing a body sensation, having an experience of orienting in the present, or an experiential moment of mastery in the play. I think about inserting BLS as a way of inviting the child to notice what is happening in the moment. Later, in treatment, when I am trying to facilitate reprocessing, I use expressions such as "let's tap on that big feeling/big thought" to create pauses in the narrative for longer and faster sets of BLS during moments of trauma digestion in the play narrative. BLS should make sense to the child as a way of calming and "looking inside" or noticing what is going on within, to whatever degree that is developmentally possible.

\section{ESSENTIAL 4: EXPANDING CAPACITY FOR STATE CHANGE}

Our emerging understanding of the neurobiological impact of trauma on the developing brains of children has a significant effect on the prioritizing of somatosensory experiences in the play therapy approach. Some children will seek this 
kind of self-regulating play on their own in the playroom, whereas others may need structure and invitation. Using a neurosequential approach (Gaskill \& Perry, 2014) helps the therapist to justify to caregivers and others why the child is "not ready to just talk about it" and why a verbal, narrative approach to trauma work is premature for many children. We seek consistent opportunities within the play to model experiences that are soothing and regulating and use repetition and cycles of excitation and modulation to "teach" the child's nervous system about calm in order to move up the neurobiological hierarchy to the more relational and reflective potential of the higher brain functions.

Within each session, either before or after (or sometimes in the midst of) the child-centered playtime, I introduce more directive miniactivities, which promote state change from distress to calm as well as generally developing resources as preparation for approaching the trauma. Games that teach and reinforce deep breathing, progressive muscle relaxation, and "bottom-up" neurobiological self-regulation are part of the play prescription for these children (see Table 1.1). Daniel Siegel (1999) first used the term "window of tolerance (WOT)" to describe the capacity of a person to remain engaged in the face of internal and external stimuli and perceived threat. Modeling and encouraging playful activities that help keep the child in the WOT is highly needed by these children. Many of these

\section{Table 1.1 Playful Activities for State Change}

\begin{tabular}{|c|c|}
\hline Breathing & Balancing and Movement \\
\hline $\begin{array}{l}\text { Deep breaths with: } \\
\text { Blowing bubbles } \\
\text { Blowing feathers } \\
\text { Blowing pinwheels } \\
\text { Blowing on imaginary butterflies } \\
\text { Blow-pens } \\
\text { Inhaling favorite scents }\end{array}$ & $\begin{array}{l}\text { Balance boards } \\
\text { Balancing peacock feathers } \\
\text { Yoga poses } \\
\text { Stretching } \\
\text { Rocking } \\
\text { Swinging } \\
\text { Playing catch }\end{array}$ \\
\hline Deep Pressure & Tactile \\
\hline $\begin{array}{l}\text { Being wrapped in a blanket } \\
\text { Being swung gently in a blanket or swing } \\
\text { Weighted items, such as stuffed animals, blankets } \\
\text { Being held gently by parent }\end{array}$ & $\begin{array}{l}\text { Slime } \\
\text { Clay } \\
\text { Wet or dry sand } \\
\text { Finger paints } \\
\text { Holding stones }\end{array}$ \\
\hline Oral & Auditory \\
\hline Gum, mints, water, snacks & Musical instruments and singing \\
\hline
\end{tabular}

This list is original to this author, but inspired by numerous sources (especially Gaskill, R., \& Perry, B. [2014]. The neurobiological power of play: Using the neurosequential model of therapeutics to guide play in the healing process. In C. Machoidi \& D. Crenshaw (Eds.), Creative arts and play therapy for attachment trauma. New York, NY: Guilford Press; Goodyear-Brown, P. (2010a). Play therapy with traumatized children: A prescriptive approach. Hoboken, NJ: Wiley; Najavits, L. M. (2002). Seeking safety: A treatment manual for PTSD and substance abuse. New York, NY: Guilford Press; Van der Kolk, B. A. (2015). The body keeps the score: Brain, mind and body in the healing of trauma. New York, NY: Penguin). 
ideas are based on sensory experiences. The play therapy literature offers many directive options compatible with these goals, including many activities within Theraplay ${ }^{\mathrm{TM}}$, and other approaches (Hong \& Mason, 2016).

Once BLS has been introduced to the child, these moments of state change offer opportunities to notice the body during short, slow sets of BLS. The key to using these very short and often only momentary interventions to gradually impact the arousal level of the child is to keep the activities novel and to use a child-responsive approach to teaching them, avoiding power struggles with the child. As Gaskill and Perry (2014) point out, the therapist should advocate with caregivers and teachers for carryover of these activities outside of the weekly therapy session for the intended results of beginning to establish new patterns within the child's arousal system.

\section{ESSENTIAL 5: EXPANDING AND ASSESSING MEMORY TARGETS IN THE POSTTRAUMATIC PLAY WITH EMDR}

Initial EMDR processing of trauma content can occur in the context of the play metaphor using the characters in the story for the initial assessment of the target and beginning reprocessing. In particular, the metaphors in the posttraumatic play may give the clinician clues about the feelings, negative cognitions, and body sensations in the memory network. Because it is difficult for children to identify negative cognitions in the ways that adolescents or adults are capable of as part of the EMDR Phase 3 assessment of the target, it is often up to the therapist to guess or suggest what negative beliefs may be stored in that network. A thoughtful consideration of the themes in the posttraumatic play can provide information about the child's sense of ongoing danger, helplessness, and self-blame.

A young client with insecure attachment stemming from her addicted mother's inconsistent care in infancy used the CCPT time to again and again reenact a similar story. She played the role of a baby figure who is alternately cared for and threatened by the imaginary characters in the room played by the therapist and various stuffed animals and toys at her direction. Over time, as she was allowed to remain in charge of the play as the director, she added more and more elements of the ambivalent attachment into the play. For example, she had the therapist act as the mother, sometimes taking care of her and feeding her, and so forth, while at other times she instructed the therapist to play the role of the mother to accuse the child of lying, to deny her the things she wanted, or to be sleeping or distracted while threats came to bother the child in the context of the developing play narrative.

Child: I'm the baby, you pretend to be the mother.

Therapist: You want me to be the mother.

Child: Yes. First you have to cover me with the blanket and feed me some of the crackers.

Therapist: Okay, baby, I am taking care of you; here is a snack for you (playfully feeds the baby the crackers one by one).

Child: Now, you have to go away and the bad guy comes. 
Therapist: Baby, I am going to go over here and . . (stage whispers: what shall I be doing?)

Child (shrugs): On your phone.

Therapist: Okay, I'm talking on my phone. . .

Child (gives therapist a puppet): Now be the bad guy.

Therapist (now has a puppet the child chose as the bad guy, and it is a scary alligator puppet): I'm coming over to the Baby now.

Child (screams): Help Mommy help!

Therapist (stage whisper): Does the mom come?

Child: No. She never hears.

This drama has many of the qualities of dynamic posttraumatic play, including urgency to the child, a sense of felt reality and emotional intensity, as well as thematic parallels to the child's own experience. In this setting, the child was able to convey the negative beliefs and emotions and body sensations experienced by the "Baby."

Therapist: That baby is feeling afraid and also alone.

Child: (Now whimpering quite realistically.)

Therapist (stepping in now with some EMDR processing): She might feel it in her heart, her stomach, her throat. . .

Child: Yes, her throat!

Therapist: Let's do a few patty cakes and see where that feeling is in her throat.

The child and therapist previously developed a "game" of using patty cakes for BLS, with a "patty cake" type of hand claps bilaterally across the line of sight (Tinker \& Wilson, 1999). During patty cake BLS, she was able to notice the distress in her own body. Jumping ahead for a moment to Essential 6, described more fully later, the self can be brought explicitly into this processing using the bridge of the moment in play, initially just momentarily until the therapist can see how much the child is ready to tolerate.

Therapist: You were a once a baby who knows what that lonely feeling is like.

Child: She thinks the Baby is already grown up.

This shift in awareness represents some important adaptive information entering the memory network. This child has uncovered in the play a moment that quite accurately represents the misattunement of her biological mother who did not consistently grasp and respond to her baby needs.

Therapist: Let's patty cake on that idea ... (BLS hand claps). See Figure 1.5 for a (nonclient) recreation of that moment in the play session. 


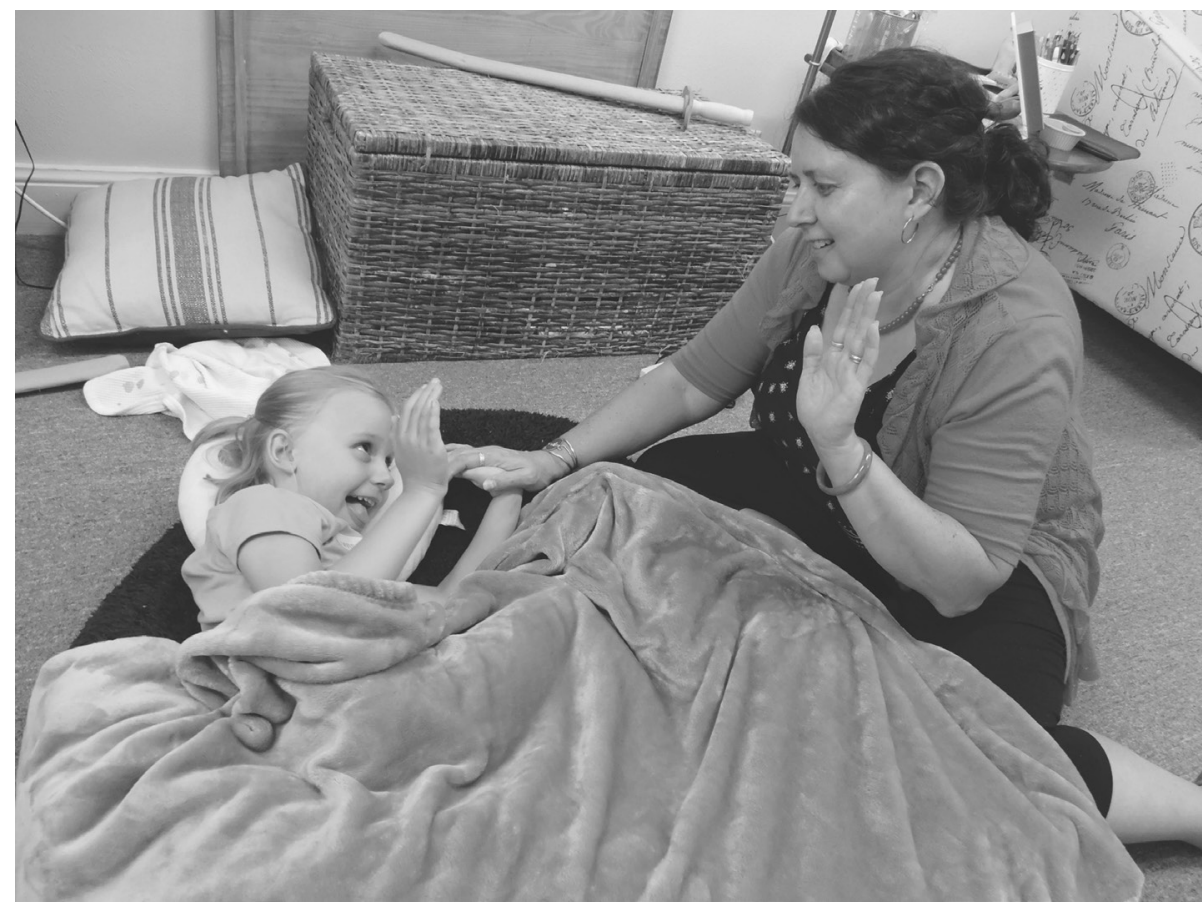

Figure 1.5 Processing Baby Memories With "Patty Cake" Bilateral Stimulation

Child: Now the mom is coming and she is mad at the bad guy - they are fighting!

(Child thrusts a foam sword into the therapist's hand.)

Therapist (hitting the alligator puppet with the sword): Like this?

Child (calming, smiling): Yes, yes!

Therapist: Take that, bad guy! Leave my baby alone! (See Figure 1.6 of therapist hitting alligator with sword.)

Child: Let's play the whole thing again.

Therapist: Patty cake with me and see how your body feels, so we can play it again (BLS).

This child could not have tolerated a standard protocol approach, and as much of the trauma was pre-verbal, she would not have had the words to convey the magnitude of her experience. Yet, through the play, she was able to not only activate that memory network but also use the opportunity to begin noticing her anger and fear in an embodied way. By using the BLS to focus her moments of noticing, the posttraumatic play remains more dynamic and within her ability to tolerate. By drawing the parallel to her own experience, the target network is more fully activated. This flexible approach to desensitization and reprocessing must be grounded in all the elements of the standard EMDR protocol, with attention 


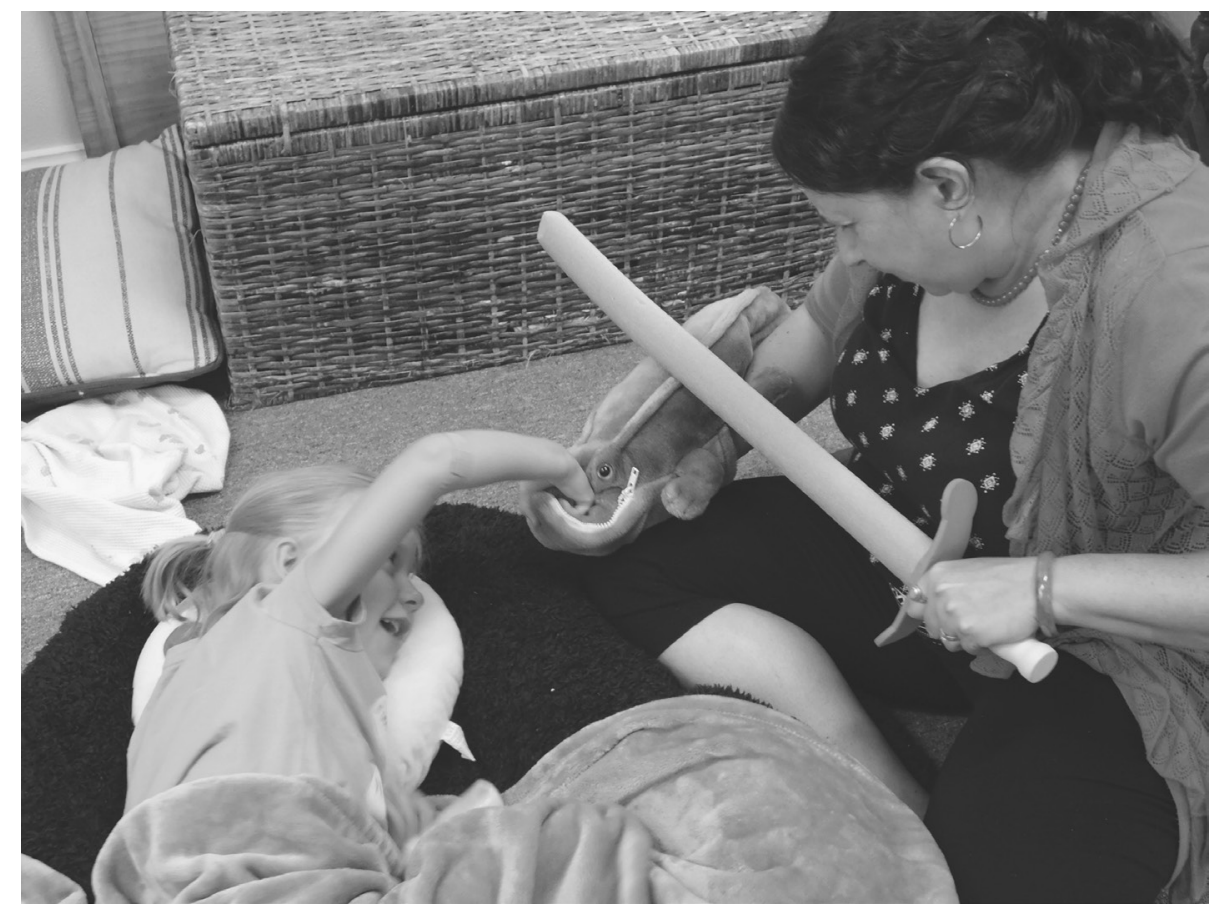

Figure 1.6 Fighting the Alligator

paid to the images, emotions, negative beliefs, and body sensations present in the network. The process is spontaneous and driven by the narrative of the play and over time may weave in and out of the child's awareness of the story as a firstperson narrative. Swimm (2018) recently published a case study in the Journal of EMDR Practice and Research in support of this kind of developmental play context for processing attachment targets.

\section{ESSENTIAL 6: BUILD BRIDGES FROM IMPLICIT TO EXPLICIT TRAUMA WORK}

When adequate relationship and preparation are in place, we do look for opportunities to build bridges of self-awareness from the play to the child's own experiences, using short episodes of EMDR processing. In these episodes, the child is invited to use BLS for "noticing" the upsetting body responses, beliefs, and so forth in a child-responsive but not highly directive way.

For example, a child who witnessed domestic violence developed a drama in the dollhouse using superheroes and villain figures. On the surface, it might seem like a fairly simplistic replay of action scenes from superhero movies. However, his persistence in frequently returning to this play when offered CCPT allowed the narrative to unfold and begin to carry meaning and content from his trauma experiences. $\mathrm{He}$ eventually expanded the story to include a helpless younger sister figure whom his hero character needed to rescue from bullying by the villain over and over again. 
Applying Essential 5, using the posttraumatic play to inform my assessment of the target memory, I could begin to recognize how this urgent narrative carried the trauma content of standing helpless while his mother was beaten and wishing to be strong enough to protect and defend her. I began developing an assessment of this trauma target over the sessions in which he returned to this narrative while still within the metaphor of the play. In this assessment, I understood that "I am helpless" was a significant negative cognition within this memory node, and the physical feeling of pent up/frozen rage was a relevant body sensation for him.

This sixth essential consideration of looking for and acting upon opportunities to bridge from the play into explicit trauma work is a challenging and perhaps novel extension of the idea of full integration of EMDR processing into the posttraumatic play. In these moments, I began by asking him to notice his own intolerance of bullying, his physical desire to defend the helpless, and accompanied these moments of noticing with BLS on drums, a favorite activity. Making the child's own self present in an explicit way offered a momentary window or bridge in which to invite him to remember his own experience of helplessness in the face of the violence and bullying directed toward his mother.

Therapist: I am noticing how much your guy hates this bully, how much anger he has...

Child (smashing his figure into the bully character): I. Hate. Him. I hate him.

Therapist: You have seen bullies too. I know you saw your Mom get bullied and hurt. Come bang on the drum with me for a minute so we can get that big feeling out, too (BLS). You wanted to save her (BLS).

Child: If I was bigger I could have. I wanted to.

Therapist: That's a big thought, let's drum on that one (BLS). Your muscles were all tight because you wanted to, but you had to keep still. (More BLS, his intensity increases.) Whew, we need a deep breath!

Child (titrates the intensity by going back into the play narrative): Leave her alone. You need to move out of this neighborhood. We do not want you!!! (Throws bully figure across the room.)

Therapist: Now he is far away from them.

Child: Yes. (To girl doll) You don't have to be afraid, he's gone.

Therapist: He is gone and it is safer now. Let's breathe. What does that feel like? Let's hug our bodies and really feel what they are feeling when he is gone (BLS with butterfly hug).

Child: I will never let any bullies hurt anyone ever again.

Therapist (reflects as a positive cognition): You want everyone to be safe now. (Butterfly hug.)

Child: He might come back next time. (Child gets up and paces across the room.)

Therapist: You and Mom are safe now, though. Let's drum on that. (Offers light up drum sticks for BLS.)

Child (drums for a few seconds, exhales): I want to play something else. 
The positive cognition is still incongruent, so he shifts uncomfortably out of processing, and I follow his lead and "allow" this distancing by responding with a CCPT reflection, instead of pushing to remain in the trauma network.

\section{Therapist: You know just what you want to do next.}

EMDR Phase 4 (desensitization and reprocessing) as described in this case example is very short, may be only 5 to 10 minutes out of an entire session. But this bite-size approach to processing is important for several reasons. In the context of this therapist-child relationship, the child has a felt sense of control over the narrative. We recognize that approaching a terrifying memory such as this one goes against the child's own instincts for self-protection, what Struik (2018) calls "waking the sleeping dogs." The children most in need of trauma processing, those with a history of chronic exposure to adversity, have a neurobiology that is very resourceful in avoiding trauma triggers. Digesting a small bite of trauma work builds distress tolerance, and over a period of time, if the therapist respects the child's movement in and out of intensity, they often are able to process bigger "bites" and sustain longer episodes of Phase 4 desensitization and reprocessing. Gradually, the child becomes occupied more fully in the role of first-person narrator of his own experience. Situating these bites of processing in the context of longer play therapy sessions also helps the child to contain the processing.

Reflecting on these brief episodes of EMDR Phase 4 (desensitization and reprocessing) situated in larger sessions of primarily CCPT can help the therapist to be ready to invite the child back into the processing toward the yet-unprocessed channels. Continuing with the boy described earlier, I recognized that the block was related to his current internal awareness of feeling safe. For him, some interweave relating to the idea and/or felt experience of the danger being truly over was needed.

When he returned in the next session to the superhero drama, I was able to use a light touch to suggest some ways in which the play could carry this needed experience of safety.

Therapist: You are able to throw that bully guy all the way across the room.

Child (repeating the action of throwing the figure again and again): You are a goner!!

Therapist: That feels really big-let's smack the drum on that (BLS). I'm wondering how to keep him from coming back? I'm wondering if any of these jails could hold him?

Child (testing the bully figure and the jails): He fits in here and I can get these dragons to guard him (see Figure 1.7). (Child visibly relaxes.)

With signs that the child is having a felt experience of safety from this interweave within the play metaphor, I bridged again to the first-person trauma memory.

Therapist: The guy who bullied you and mom is in a jail like this I bet. Let's hit the drum while we notice how that feels (BLS). 


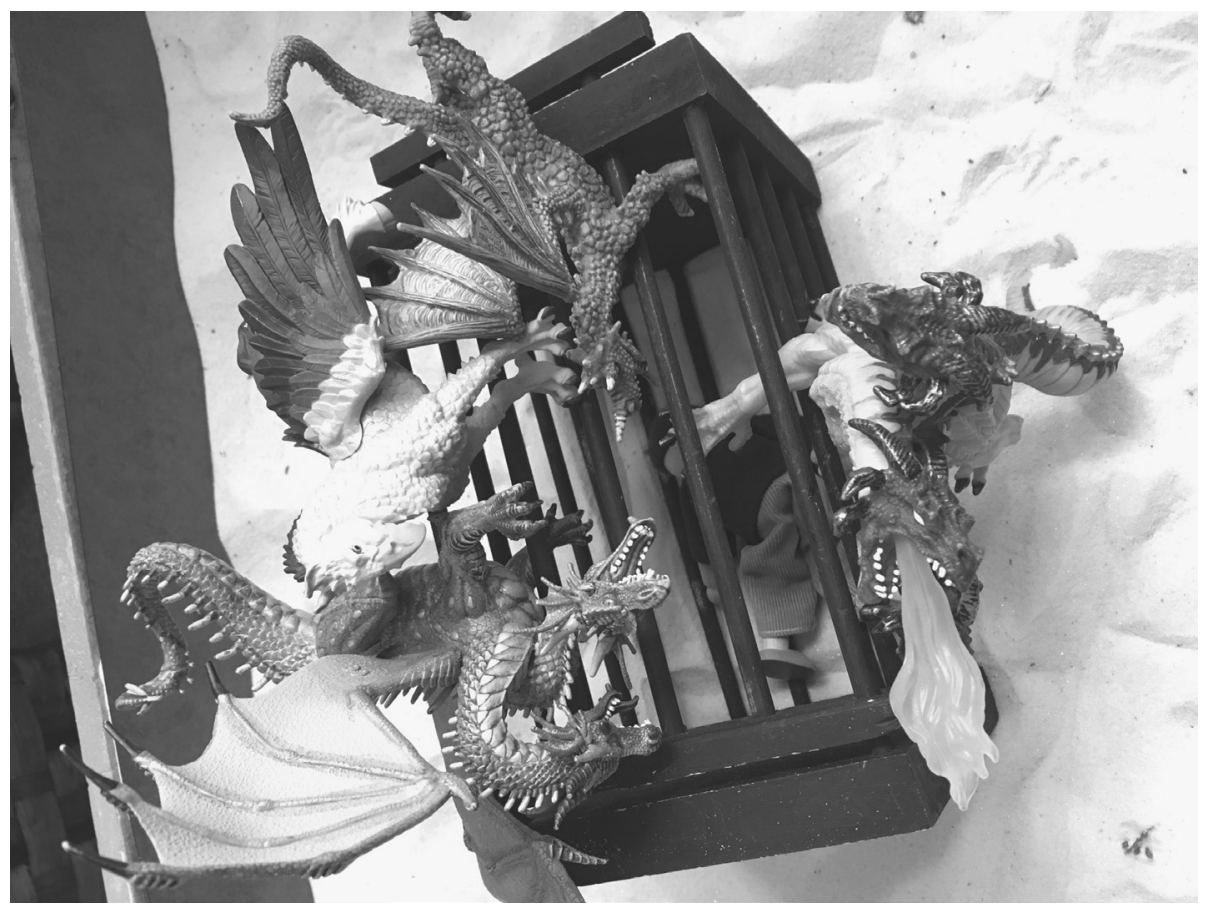

Figure 1.7 Mastery of Threat: "The Bully Is in Jail"

Child (laughing): No dragons though. Policemen. (Puts a toy policeman on the pile.)

Therapist: They are safe now.

Child (accepts positive cognition for the first time): Safe . . yeah . . for now.

Therapist: Let's do that butterfly hug and see what that safe feeling is like in our bodies.

For clinicians with experience in using the standard EMDR protocol with children, there is an all-too familiar sense that we are not always really in the memory network when we ask kids to bring up the disturbing images. Processing ends up feeling superficial. As we move through the Phase 3 assessment questions from the standard protocol, the child might shrug or answer "I don't know." When children are engaged in posttraumatic play such as this example illustrates, which explicitly or metaphorically reenacts elements of the trauma experience, we are more fully in the trauma network of associations and more likely to help them take the "right bites" that will help the memory shift. When the therapist uses the light touch of a play therapy reflection to try out a possible positive or negative cognition or interweave that might or might not fit, the child can go back into the play as needed without getting overwhelmed. 


\section{ESSENTIAL 7: ONGOING REEVALUATION OF MEMORY TARGETS}

How much reprocessing is enough? As challenging as it is to answer this question in trauma work with adults, it is even harder to ascertain whether there is remaining distress in the memory network based on a child's self-report. Because it is quite challenging for young children to use the Subjective Units of Disturbance Scale accurately, and they tend to report everything as either a 10 or 0 , we must rely more heavily on the external evidence such as through the use of caregiver interviews for Phase 8 reevaluation of targets (Beckley-Forest, 2015; BeckleyForest \& Monaco, 2016). We can ask the caregivers to keep track of the child's moods, eating and sleeping patterns, any nightmares, or improvement of functioning. The therapist should continue to return to processing in small chunks as needed as well as attending to the shifts in the child's play themes as a clue about the remaining distress.

In the case of the boy described earlier, the superhero play narrative continued to orbit similar themes of helplessness and the desire to rescue over many sessions. I was able to help him and his mother develop some increased resources around felt safety, then-and-now time orientation, and helping her to reflect on how helplessness had remained a trigger for episodes of rage for him. As we continued to bridge between the play metaphor and his own story, we were able to move toward a more explicitly first-person narrative about the trauma, and the reduction of his distress became more apparent. In the play metaphor, the boy began on his own to incorporate more adaptive solutions to the dilemmas of the characters, for example, creating an elaborate courtroom scene, before losing interest in the story altogether. With this shift in the play, accompanied by remission of some symptoms, we can feel more certainty that the target memory is no longer as distressing to him. In this case, as the play themes became less important to him, his night fears decreased and he began sleeping in his own bed.

In the case of very early trauma, such as pre-verbal attachment experiences, some younger children may never really engage in extensive explicit trauma work around events that "happened to $\mathrm{me}^{\prime}$ as they have great trouble connecting to these events. Instead, we would look for the shifts in intensity, interest, and content of the play themes. Also, due to their emerging development of abstract thought, some children who have done significant trauma digestion through play therapy in earlier childhood may need return episodes of processing as they mature and begin to ask questions that were not available to them in the earlier episode of treatment. An example of this would be a young child who does some processing of an experience of sexual abuse but may need a return to treatment in puberty as an emerging awareness of age-appropriate sexual expression begins to develop.

\section{ESSENTIAL 8: INVOLVING PARENTS THROUGHOUT TREATMENT}

The final of these eight essential considerations is the most complicated to apply. Every child therapist has to explore their own theoretical orientation and countertransference around how and when to integrate parents into children's treatment. The context for these decisions is further complicated by children who have not had consistent caregivers, such as foster children who have had multiple 
placements or children with parents whose capacity for attachment and support is compromised by their own trauma or attachment history. Therapists may struggle to find ways to include parent figures in the safe therapeutic alliance and may have ambivalence about them based on the nature of the child's trauma, the parents' own role in failing to adequately protect the child, and the impairments in the parental attachment pattern, which are negatively impacting the child. Despite the complexity, the literature is clear that parents (by which we also mean other caregivers including foster and adoptive parents, and grandparents; even possibly extending to residential direct care workers in some cases) must be involved in therapy as a resource and for fullest benefit of trauma processing with EMDR (Gomez, 2012; Lovett, 1999; Wesselman et al., 2014). Likewise, a significant factor in the research that established the evidence base for play therapy was the finding that the benefits of play therapy were markedly enhanced when parents were fully involved in treatment (Lin \& Ray, 2015).

CPRT is an example of a filial approach within play therapy, which attempts to extend the attuned relationship that the play therapist has with the child to the parent-child system (Landreth \& Bratton, 2006). In the play therapy environment, the parent and child have the opportunity to experiment more flexibly with attunement in their social engagement with one another. The child has the opportunity to be enjoyed and fully seen by the parent, increasing what Fonagy, Gergely, Jurist, and Target (2018) called mentalization, or the parent's ability to "hold another's mind in mind." Whenever possible, we try to model and coach parents to enter the play therapy space with their child and assist them in expanding their capacity to witness and notice their child and their play. When parents have the capacity to be child-centered even for short periods of time, they are encouraged in this role, as it will promote an expanded capacity for mentalization over time.

We know through the work of Stephen Porges on the polyvagal theory that trauma and its aftermath tend to disengage the social engagement system in response to threat. Chronic trauma represents an ongoing suppression of the brain's own ability to form and sustain attachments and regulate the neurobiology in the face of moment-to-moment challenges. The problematic symptoms in children described by their parents as the impetus for treatment can often be explained as failures of their social engagement system (Porges, 2011).

The attachment trauma of the parent may be echoed in the attachment disruptions the child is experiencing, and the child's posttrauma symptoms may trigger traumatic material for the parent (Gomez, 2013). Parallel comprehensive treatment for the parent-child dyad must of necessity be a component of healing, and there are models for this in both the EMDR therapy and play therapy literature. The Integrative Attachment Trauma Protocol (Wesselman et al., 2014) incorporates family therapy and EMDR processing in a multimodal, multitherapist approach. Naturally, the child therapist is often in the position of wishing the parent was engaging in their own trauma therapy. Even when involved with the family system as a single practitioner, there are treatment plan benefits from conducting a thorough assessment of the parent's own attachment pattern (consider tools such as the Adult Attachment Interview [George, Kaplan, \& Main, 1996], or the Family Experience in Childhood Scale [Gonzalez, Mosquera, \& Leeds, 2011]) to help the therapist make decisions on how to involve the parent in the child's play therapy and how much structure the parent will need to be successful 
and recommendations to parents about how their own attachment needs may be impacting the child.

For example, some years ago, I worked with an adoptive mother and the son she adopted through the foster care system using a filial approach to play therapy. This mother was coached on the basic rules of reflecting and responding to the child's imaginative play, primarily on how to stay connected through observations instead of asking or answering questions, teaching, or influencing the play. Over several sessions she watched as I modeled these responses, gradually taking over this role while I receded to more of an observer or facilitator role. One of the greatest difficulties for parents in play therapy is to avoid coaxing the child to be more prosocial (such as by saying-“'Oh, we shouldn't hit the baby doll like that, we should play nice"), which has the effect of shutting down the often-intense posttraumatic themes in the play. This mother found the intensity of the child's play dramas with themes of abandonment and rage to be upsetting at times, but she made good use of support from me and from her own therapist and partner and so was able to remain present to the child and play out the narratives as the child directed them. This is the type of parent who might be able to be present throughout the various phases of treatment and enhance the therapeutic safety of the play.

It is not always advisable to have parents present in each play therapy session. In particular, when the content of the child's play has the potential to carry the posttraumatic content, as described earlier (Essentials 5 and 6) and when the therapist is trying to hold the space for this play and expand it through a childcentered approach, parents may not be ready to collaborate, as they may be too triggered themselves. Often children's play is quite different when parents are in the room and the child may be trying, whether consciously or unconsciously, to protect the parent from their distress. There may be implied cognitive distortions around shame and blame that the therapist or the parent has not yet addressed.

Another case example serves to illustrate when it might be a good idea to limit the presence of the parent. In this case, the trauma history for the child was parallel to that of the mother, as there had been several incidents of domestic violence between the parents. This very committed and attuned mother had taken all the steps to get her and her young child to safety, but the loss of regular contact with the father had a very different meaning for her than for the child, and her presence in the play therapy room during play therapy and trauma digestion that included themes of missing his father might have created loyalty binds for him. In this case, I chose not to have her in the room for the CCPT or explicit trauma work, but she was still very actively engaged in the therapy in learning coping skills as described later.

When using games and play strategies to promote state change (see Essential 4), we want parents to be playing and learning alongside their children. Theraplay is an example of a directive play therapy approach to recreating or enhancing basic relationship engagement between the parent and child in a context that helps children to oscillate between excitement and calm, while promoting engagement with the parents and the child's acceptance of nurturing, structure, and challenge. The Theraplay approach is planful and directive and relies on repetition and systematic modeling and feedback to the parent delivered on a moment-to-moment basis. It is associated with positive outcomes in the interaction patterns in the 
parent-child dyad (Jernberg \& Booth, 2001). Theraplay and EMDR are highly compatible approaches, and the full integration of these approaches is well established among therapists who are trained in both (Gomez \& Jernberg, 2012).

An educational approach that helps parents understand their child's (sometimes quite narrow) WOT and how to use playful coregulation to expand this window and reestablish calm is invaluable to parents and other caregivers. I limit problem talk in front of the child and try to help parents shift from a solely causeand-effect behavioral approach to one based on an understanding of the hyperand hypoaroused states and their child's threat response system. I use playful engagement of the parent in coregulating their child with lots of repetition and practice. Activities that were described in Essential 3 earlier should include the parent or other caregivers such as prop-based ways of breathing (e.g., feathers, pin wheels, bubbles), using soothing sensory input (e.g., lotions, essential oils, relaxing music), tastes (e.g., cold drinks, mints, ice, small snacks), and physical activity (e.g., large muscle activity, deep pressure, nature). These game-based "experiments" are offered as an adjunct to the main body of the play therapy sessions while treatment is getting established, often as rituals in the opening or closing of the session, and help the parent and child increase their repertoire of statechange activities. Empowering parents to become coregulators of their children has important benefits in carrying over in-session experiences and can help with the stabilization that supports progress in therapy, especially if we want children to risk venturing into trauma content. Goodyear-Brown (2010a) describes numerous additional activities to encourage this kind of coregulation. Engaging families in this kind of play offers moments to install shared delight as adaptive information using slow, short sets of BLS. For example, I have had parents and children tap using the butterfly hug while laughing at something together, rock back and forth while mirroring each other's facial expressions, and adding a brief set of eye movements or tapping to a moment when the child is enjoying being held/rocked like a baby by the mother.

Each child's progress through therapy demands an ongoing individualized assessment of how best to involve parents as a resource. As a general rule, when the traumatic elements of the memories are still in the metaphor of the play, I am more cautious about having parents witness this play unless they have shown they are able to attune and contain their own reactions to a sufficient degree as not to compromise the emotional safety the child needs to expand these themes and enter the traumatic memory network. As digestion of the trauma moves toward the more explicit first-person narratives, I am more likely to invite the child to include the parent as a way of not only integrating the experience, but also potentially using the parent as a source of adaptive information and interweaves. Including the parent necessitates preparation sessions with the parent to help them to digest their own emotional reactions sufficiently to be present to the child without overreacting or oversoothing. Lovett (1999) describes a step-by-step approach to preparing parents in using a storytelling approach to EMDR processing of targets with children, and this can be enhanced through the addition of playful props for acting out the story. The investment of time in the relationship with the parent is worth it when we see the shifts in the day-to-day interactions between the parent and child that are indicative of increased emotional safety as we move through the phases of EMDR treatment. Also, caregiver input is needed 
in order to evaluate the success of both the EMDR protocol and play therapy interventions as discussed in Essential 7.

\section{POTENCY OF INTEGRATED MODELS OF TREATMENT IN COMPLEX POSTTRAUMATIC STRESS DISORDER AND DISSOCIATION}

Therapists who work with children, especially children with complex, early trauma, are well aware that even the best, most developmentally accessible interventions will not offer a quick fix to the traumatized child and their wounded nervous system. My efforts to articulate the urgency of a sophisticated use of play therapy as the best context for EMDR have been significantly influenced by Knipe's concept of the Constant Installation of Present Orientation and Safety, or CIPOS (2018). Knipe's emphasis on helping clients with dissociative symptoms establish and maintain continual here-and-now orientation led me to think more deeply about how play therapy serves as the best way of delivering CIPOS to younger children. The attunement and acceptance delivered via reflective feedback of a trained play therapist in the presence of posttraumatic play is a form of child-friendly CIPOS and over time will help child clients build capacity for desensitization to trauma content using EMDR by an accumulated sense of felt safety in the here-and-now experience of the therapy room.

For the most vulnerable and damaged children, innovation and integration are essential to cope with the intensity of their symptoms, in particular the managing and healing of dissociative states. A full clinical literature to address structural dissociation in children is still emerging. Frances Waters's landmark book, Healing the Fractured Child (2016), resonated deeply with every clinician who has spent considerable time with traumatized children. The incredible potential of applying classic structural dissociation theory in a practical and integrative way in child therapy settings is still being unpacked and realized. Using the lens of structural dissociation theory to comprehend the intense experiences of posttraumatic play in the therapy room generates even more questions than answers. Obvious opportunities to use creative and dramatic play to recognize and work with the child's dissociative self-states represent an amazing frontier for further integration of play therapy as the setting for this particular kind of trauma work, such as Monaco's use of a playroom metaphor as a "conference room" for a child's self-states to meet and initiate cooperation (Beckley-Forest, Goodyear-Brown, \& Monaco, 2018).

\section{CONCLUSION}

In any blending or integrating of models, fidelity to the original and purest version of the contributing models must be an important consideration. CCPT, other models of play therapy, and the EMDR standard protocol each have processes and procedures developed over time, and the evidence that supports their efficacy is based on a faithful adherence to their application under controlled circumstances. 
Adler-Tapia and Settle (2008), while articulating the difficulties of manualizing EMDR with young children, found that "children were much less likely to produce all the phases of the protocol verbally but instead were successful with expressive techniques, including play therapy and art therapy. Offering alternative methods for expression did not preclude the therapist from demonstration of fidelity to the eight phases" (p. 105).

When research-supported models of care are modified or adapted in any way, we must do so with intention and purpose and engage in a continual evaluation of the benefits to the client, ideally with the help of good clinical consultation. We must also construct sequences of treatment experiences with an eye on the neurobiology of posttrauma and make continually attuned adjustments based on the child's felt experience of safety. Eliana Gil (2016) speaks to a clientcentered approach to treatment integration when she states that "posttraumatic play advances therapy goals and prepares children for specific techniques such as Trauma-Focused Cognitive Behavioral Therapy (TF-CBT) and EMDR" (p. 19).

High-quality research, which can offer more information on how best to harness the clinical benefits of integrating specific models of play therapy and EMDR, would improve the efficiency of such an endeavor, but until such time as that specific research emerges, we must use the best clinical instincts of highly trained child therapists as the guide for how to best integrate these evidencebased approaches. With that in mind, the next steps in fuller integration must include increasing the numbers of therapists trained and credentialed in both play therapy and EMDR, and those qualified to provide specialized consultation in both fields, in order to respond with skill and flexibility to the complex needs of the children.

\section{REFERENCES}

Adler-Tapia, R., \& Settle, C. (2008). EMDR and the art of psychotherapy with children: Treatment manual. New York, NY: Springer Publishing Company.

Adler-Tapia, R., \& Settle, C. (2016). EMDR and the art of psychotherapy with children (2nd ed.). New York, NY: Springer Publishing Company.

Axline, V. (1964). Dibs in search of self. New York, NY: Random House.

Badenoch, B. (2008). Being a brain-wise therapist: A practical guide to interpersonal neurobiology. New York, NY: W. W. Norton.

Beckley-Forest, A. (2015). Play therapy and EMDR: A conversation. Play Therapy, 10(3), 10-14.

Beckley-Forest, A. (2019). Exploring the intersection of EMDR and play therapy. Go With That, 24(1), 7-11.

Beckley-Forest, A., Goodyear-Brown, P., \& Monaco, A. (2018, October). EMDR and play therapy: A powerful combination. Presentation at the 23rd EMDR International Association Conference, Atlanta, GA.

Beckley-Forest, A., \& Monaco, A. (2016, August). Teaching kids to play EMDR therapy. Presentation at the 21st EMDR International Association Conference, Minneapolis, MN.

Bratton, S., Ray, D., Rhine, T., \& Jones, L. (2005). The efficacy of play therapy with children: A meta-analytic review of treatment outcomes. Professional Psychology: Research and Practice, 36, 376-390. doi:10.1037/0735-7028.36.4.376 
Brody, V. A. (1997). The dialogue of touch: Developmental play therapy (2nd ed.). Northvale, NJ: Jason Aronson.

Chen, Y. R., Hung, K. W., Tsai, J. C., Chu, H., Chung, M. H., Chen, S. R., . . Chou, K. R. (2014). Efficacy of eye-movement desensitization and reprocessing for patients with posttraumatic-stress disorder: A meta-analysis of randomized controlled trials. PLoS One, 9(8), e103676. doi:10.1371/journal.pone.0103676

De Roos, C., Greenwald, R., den Hollander-Gijsm, M., Noorthoorn, E., van Buuren, S., \& de Jongh, A. (2011). A randomized comparison of cognitive behavioral therapy (CBT) and eye movement desensitization and reprocessing in disaster-exposed children. European Journal of Psychotraumatology, 2, 5694-5704. doi:10.3402/ejpt.v2i0.5694

Fleming, J. (2012). The effectiveness of eye movement desensitization and reprocessing in the treatment of traumatized children and youth. Journal of EMDR Practice and Research, 6( 1). 10.1891/1933-3196.6.1.16

Fonagy, P., Gergely, G., Jurist, E., \& Target, M. (2018). Attachment and reflective function: Their role in self-organization. In P. Fonagy, G. Gergely, \& E. Jurist (Eds.), Affect regulation, mentalization and the development of the self (pp. 21-63). New York, NY: Routledge.

Gaskill, R., \& Perry, B. (2014). The neurobiological power of play: Using the neurosequential model of therapeutics to guide play in the healing process. In C. Machoidi \& D. Crenshaw (Eds.), Creative arts and play therapy for attachment trauma. New York, NY: Guilford Press.

George, C., Kaplan, N., \& Main, M. (1996). Adult attachment protocol (3rd ed.). Unpublished manuscript, Department of Psychology, University of California at Berkeley.

Gil, E. (2006). Helping abused and traumatized children: Integrating directive and non-directive approaches. New York, NY: Guilford Press.

Gil, E. (2016). Posttraumatic play in children: What clinicians need to know. New York, NY: Guilford Press.

Gomez, A. M. (2012). EMDR therapy and adjunct approaches with children: Complex trauma, attachment and dissociation. New York, NY: Springer Publishing Company.

Gomez, A. M., \& Jernberg, E. (2012). Using EMDR therapy and theraplay. In A. M. Gomez (Ed.), EMDR therapy and adjunct approaches with children: Complex trauma, attachment and dissociation. New York, NY: Springer Publishing Company.

Gonzalez, A., Mosquera, D., \& Leeds, A. (2011). Family experiences in childhood scale. Retrieved from https://docplayer.net/147066497-Family-experiences-in-childhood-scale-fecs.html

Goodyear-Brown, P. (2010a). Play therapy with traumatized children: A prescriptive approach. Hoboken, NJ: Wiley.

Goodyear-Brown, P. (2010b). The worry wars. Nashville, TN: Author.

Goodyear-Brown, P. (2019). Trauma and play therapy. New York, NY: Routledge.

Greenwald, R. (1999). EMDR in child and adolescent psychotherapy. Northvale, NJ: Jason Aronson.

Homeyer, L., \& Sweeney, D. (2016). Sandtray therapy: A practical manual (3rd ed.). New York, NY: Routledge.

Hong, R., \& Mason, C. M. (2016). Becoming a neurobiologically informed play therapist. International Journal of Play Therapy, 25(1), 35-44. doi:10.1037/pla0000020

Jarero, I. (2002). The butterfly hug: An update. EMDRIA Newsletter, 7(3), 6.

Jernberg, A., \& Booth, P. (2001). Theraplay. San Francisco, CA: Jossey-Bass.

Kemp, M., Drummond, P., \& McDermott, B. (2010). A wait-list controlled pilot study of eye movement desensitization and reprocessing (EMDR) for children with post-traumatic stress disorder (PTSD) symptoms from motor vehicle accidents. Journal of Clinical Child Psychology and Psychiatry, 15(1), 5-25. doi:10.1177/1359104509339086 
Knell, S. M. (2009). Cognitive behavioral play therapy: Theory and applications. In A. A. Drewes (Ed.), Blending play therapy with cognitive behavioral therapy: Evidence-based and other effective treatments and techniques (pp. 117-133). Hoboken, NJ: Wiley.

Knipe, J. (2018). EMDR toolbox: Theory and treatment of complex PTSD and dissociation (2nd ed.). New York, NY: Springer Publishing Company.

Kottman, T. (2013). Partners in play: An adlerian approach to play therapy (3rd ed.). Alexandria, VA: American Counseling Association.

Landreth, G. (2012). Play therapy: The art of the relationship (3rd ed.). New York, NY: Routledge.

Landreth, G., \& Bratton, S. (2019). Child parent relationship therapy (CPRT): A 10 session filial model (2nd ed.). New York, NY: Routledge.

Lin, Y., \& Bratton, S. C. (2015). A meta-analytic review of child-centered play therapy approaches. Journal of Counseling and Development, 93(1), 45-58. doi:10.1002/j.1556-6676 .2015.00180.x

Lovett, J. (1999). Small wonders: Healing childhood trauma with EMDR. New York, NY: Free Press.

McGuiness, V. (2003). Integrating play therapy and EMDR with children. Bloomington, IN: AuthorHouse.

Moreno-Alcazar, A., Treen, D., Valiente-Gomez, A., Sio-Erlos, A., Perez, V., Amann, B. L., \& Radua, J. (2017). Efficacy of eye movement desensitization and reprocessing in children and adolescents with post-traumatic stress disorder: A meta-analysis of randomized controlled trials. Frontiers in Psychology, 8(1750), 1-10. doi:10.3389/fpsyg.2017.01750

Najavits, L. M. (2002). Seeking safety: A treatment manual for PTSD and substance abuse. New York, NY: Guilford Press.

Porges, S. (2011). The polyvagal theory: Neurophysical foundations of emotions, attachment, communication and self-regulation. New York, NY: W. W. Norton.

Ray, D., Armstrong, S., Balkin, R., \& Jayne, K. (2015). Child centered play therapy in the schools: Review and meta-analysis. Psychology in the Schools, 52, 107-123. doi:10.1002/ pits. 21798

Schaefer, C. (2011). Prescriptive play therapy. In C. Schaefer (Ed.), Handbook of play therapy (pp. 365-377). Hoboken, NJ: Wiley.

Schaefer, C., \& Drews, A. (2014). Therapeutic powers of play: 20 core agents of change. Hoboken, NJ: Wiley.

Shapiro, F. (2017). Eye movement desensitization and reprocessing (EMDR) therapy, third edition: Basic principles, protocols and procedures. New York, NY: Guilford Press.

Siegel, D. (1999). The developing mind: How relationship and the brain interact to shape who we are. New York, NY: Guilford Press.

Struik, A. (2018). The sleeping dogs method to overcome children's resistance to EMDR therapy: A case series. Journal of EMDR Practice and Research, 12(4), 224-241. doi:10.1891/1933-3196.12.4.224

Substance Abuse and Mental Health Services Administation. (2012). Comparative effectiveness research series: Eye movement desensitization and reprocessing therapy: An information resource. SAMHSA's National Registry of Evidence-based Programs and Practices. Retrieved from https://cdn.ymaws.com/www.emdria.org/resource/resmgr/research /treatment_guidelines/samhsa.2012.nrepp-comparativ.pdf

Swimm, L. (2018). EMDR Intervention for a 17-month-old child to treat attachment trauma: Clinical case presentation. Journal of EMDR Practice and Research, 12(4), 269-279.

Swinden, C. (2018). The Child-centered EMDR approach: A case study investigating a young girl's treatment for sexual abuse. Journal of EMDR Practice and Research, 12(4), 282-295.

Terr, L. (1990). Too scared to cry. New York, NY: Basic Books. 
Tinker, R. H., \& Wilson, S. A. (1999). Through the eyes of a child: EMDR with children. New York, NY: W. W. Norton.

Van der Kolk, B. A. (2015). The body keeps the score: Brain, mind and body in the healing of trauma. New York, NY: Penguin.

Waters, F. (2016) Healing the fractured child: Diagnosis and treatment of youth with dissociation. New York, NY: Springer Publishing Company.

Wesselman, D., Schweizer, C., \& Armstrong, S. (2014). Integrative team treatment for attachment trauma in children. New York, NY: W. W. Norton. 